\title{
Diffuse Reflectance Imaging Differentiates Healing from Non- Healing Wounds in Diabetic Foot Ulcers
}

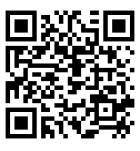

\author{
Suset Rodriguez BS ${ }^{1}$, Jiali Lei BS ${ }^{1}$, Elizabeth Solis BS ${ }^{1}$, Katrina Epnere MS $^{1}$, Francisco Perez Clavijo DPM${ }^{2}$, \\ Steven Wigley DPM${ }^{3}$ and Anuradha Godavarty $\mathrm{PhD}^{* 1}$
}

${ }^{1}$ Optical Imaging Laboratory, Department of Biomedical Engineering, Florida International University, Miami, Florida, USA

${ }^{2}$ Podiatry Care Partners Inc., Doral, Florida, USA

${ }^{3}$ Wigley Foot and Ankle LLC, North Miami, Florida, USA

Received: May 28, 2018; Published: June 07, 2018

*Corresponding author: Anuradha Godavarty, PhD, Optical Imaging Laboratory, Department of Biomedical Engineering, Florida International University, Florida, USA

\begin{abstract}
Diabetic foot ulcers (DFUs) are the most common of the plethora of complications arising from diabetes. To date, clinicians employ visual inspection of the wound site during its standard 4- week healing process via monitoring of surface epithelization. Herein, a hand-held nearinfrared optical scanner (NIROS) has been developed for non-contact imaging of DFUs and differentiating between healing and non-healing ulcers based on differences in blood flow to the wound and its surroundings. Non-contact near-infrared area imaging of DFUs have been carried to obtain diffuse reflectance images of the wound and its surroundings. These images were used to quantify optical contrasts between the wound and its surroundings. The variability in imaging conditions, analysis and operator dependency were assessed to determine the robustness of the imaging approach. All experimental studies were carried out at two podiatric clinics. Seven healing and three non-healing DFUs were imaged using NIROS. Optical contrast of wound: background was estimated from diffuse reflectance images to differentiate healing from non-healing DFUs. Optical contrast was distinctly different for healing (positive contrast) and non-healing (negative contrast) wounds, independent of the varying imaging and data analysis conditions. The application of a portable hand-held imager to assess the healing or nonhealing nature of DFUs during weekly clinical treatment is significant since physiological changes, as observed using NIROS, manifest prior to visual reduction in wound size during the healing process.
\end{abstract}

Keywords: Near-Infrared Imaging; Diabetic Foot Ulcers; Optical Contrast; Diffuse Optical Imaging; Hand-Held; Diffuse Reflectance Imaging

\section{Introduction}

\section{Diabetic Foot Ulcers}

Foot ulcerations are the most common of the plethora of complications arising from diabetes. In the United States alone, 29.1 million people have diabetes, and about $60 \%$ of non- traumatic amputations of the lower limbs are performed on diabetic adult with foot ulcers [1]. The treatment of diabetic foot ulcers (DFUs) costs \$ 9-13 billion annually [2], causing significant economic, clinical, and emotional burden on these patients [2-10]. About $84 \%$ of lower extremity amputations due to diabetes begin with foot ulceration [4-6]. The health care cost are driven by interventions to prevent ulcer, development of DFU care plan to shorten healing-time, management plan to prevent amputation, and management plan for the disabled after amputation. Clinicians have been struggling to determine effective methods to predict onset of ulcer or optimize treatment plan to shorten healing time.

\section{Wound Healing Assessment}

According to the Wound Healing Society (WHS), the accepted standard of improvement is 4-week, by when a reduction of the wound size by $40 \%$ is expected from the treatment [11-13]. If it extends beyond 4-weeks, re-evaluation of wound and other treatment options are considered. It has been suggested that up to $85 \%$ of amputations can be avoided when an effective care plan is adopted, with one of them being effective local wound care [14-16]. To date, clinicians employ visual inspection of the wound site during its standard 4-week healing process via monitoring of surface epithelization. More specifically, the wound area is measured directly using several different ways. These include tracings inked on clear acetate, calibrated digital photographs, or direct measure of the maximum length and width of the wound [16]. The criterion most accepted for healing studies is complete closure or epithelialization after 20 weeks of treatment [17-18]. In some cases a reduction in 
wound area at 10 and 20 weeks is also used. However, visual inspection for surface epithelization is not an implication of internal healing in many cases. If left untreated immediately, the extent of leg/foot amputation may increase due to lack of response to the treatment. Researchers in the past expressed that non- invasive assessment for such vascular diseases requires other tests such as absolute ankle pressure (unlike ankle-brachial pressure index that is unreliable) [19], toe pressure measurements, color duplex ultrasonography [20] and/or measurements of transcutaneous oxygen (especially around the wound) [21]. However, these techniques are not performed during periodic weekly assessment and treatment of wounds. To date, the clinical gold standard of wound healing monitoring on a periodic basis has been visual assessment of the wound, with no imaging intervention of the wound site and its sub-surface to monitor the internal wound healing process.

\section{NIRS for Wound Imaging}

Near-infrared spectroscopy (NIRS) is an emerging non-invasive and non-ionizing technology that can map the changes in hemodynamics in the site of interest (e.g. the wounded and healthy tissue site) even up to a few centimeters deep. The technology uses near-infrared (NIR) light between 650-1000 nm to image deep tissues in order to map the spatial and temporal distribution of the optical properties (which translate to the oxy- and deoxy-hemoglobin changes) during repeated visits of the treatment process. Researchers have implemented NIRS towards wound healing studies in animals and pilot human subject studies as well [12,13,22-35]. Papazoglou and her group demonstrated that near-infrared spectroscopy (NIRS) can predict wound healing in human diabetic foot ulcers [12,13,24,25,29-32]. The developed NIR optical device by Papazoglou's group demonstrated its efficacy in monitoring blood flow changes during wound healing process and differentiated a healing and a non-healing foot ulcer in diabetic patients [24,25,2932]. The device is based on NIR spectroscopic information obtained at six discrete point locations using optical fibers that contact the wound site and the surrounding normal site during imaging studies performed over a 20-week period. Their studies stated that optical absorption in a healing wound initially diverges from absorption in a healthy tissue, and later converges with the absorption of healthy tissues as the wound almost heals. The hemoglobin concentrations successfully distinguished healing from non-healing ulcers, and reduction of hemoglobin in the wound site and approaching that of the normal surrounding tissue is indicative of wound healing. Recently a hand-held non-contact based area imaging near- infrared optical scanner (NIROS) has been developed in our Optical Imaging Laboratory. The optical scanner has been recently used to demonstrate real-time dynamic imaging of the changes in blood flow in the dorsal of the hand during venous occlusion [26,27]. A pilot study demonstrated that NIROS could differentiate healing and non-healing lower extremity ulcer $[34,35]$. Herein, imaging studies were performed on 10 diabetic foot ulcers (healing and non-healing) in order to determine its ability to assess healing or non-healing consistently, under various imaging and analysis conditions. Pilot studies were performed on two DFU cases to map hemodynamic changes in and around the wounds.

\section{Materials and Methods}

\section{Instrumentation}

A near-infrared optical scanner (NIROS) was employed to perform non-contact imaging over large tissue surfaces $\left(\sim 35 \mathrm{~cm}^{2}\right)$. The clinical set-up of NIROS for wound imaging is shown in Figure 1. The imaging system uses LED-based source, an NIR sensitive camera (IDS, Germany) mounted on an articulating arm that allows flexible movement of the device to image any location of the wound in the lower extremities. A NIR LED ((830-nm) is used in the current studies to illuminate near-infrared light onto the tissues. The LED light source is driven by an LED driver, which controls the radiating power of the LED to a maximum of $50 \mathrm{~mW}$ each. The LED driver and the NIR sensitive camera are synchronized during data acquisition via a micro controller unit (F28027, Texas Instruments, USA). The entire device is powered by the computer's USB port $(5 \mathrm{~V}$, maximum $900 \mathrm{~mA}$ ) without involving any external outlets. NIR light at launched onto the tissue surface as a continuous-wave in a non-contact reflectance imaging mode (with optical power $<5 \mathrm{~mW}$ ). The diffuse reflected NIR signals from the tissue surface is detected by the camera after the signals have been filtered using a longpass optical filter. A Matlab-based imaging software is developed in order to automate the data acquisition process such that dynamic NIR images are captured at $10 \mathrm{~Hz}$ frame rate by the NIR detector and saved to the computer. An endoscopic camera (Oasis Scientific, USA) is used to acquire digital white light (or visible light) images of the wound apart from NIR images obtained using NIROS.

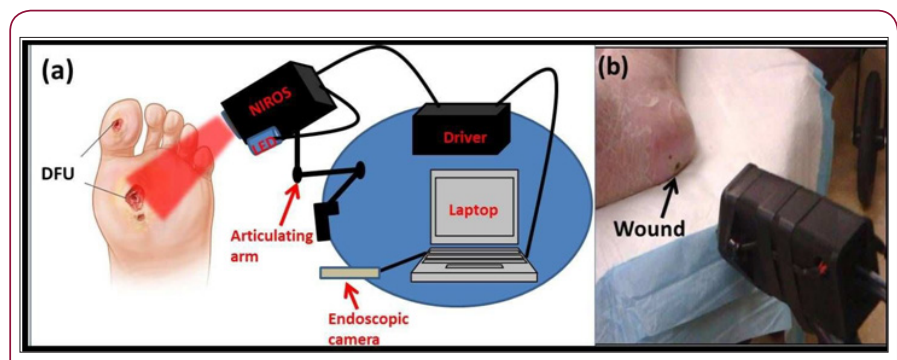

Figure 1: (a) Schematic of the near-infrared optical scanner (NIROS) attached to an articulating arm and controlled by a laptop, along with an endoscopic camera for obtaining digital white light images, (b) Non- contact imaging of wounds (here it is DFU) using NIROS.

\section{Subject Recruitment}

Non-contact optical imaging studies were carried on diabetic subjects with DFUs (healing or non-healing). A total of 3 subjects with one or more DFUs (total 10 wounds) and between the ages of 41-70 years participated in this Florida International University's IRB approved study (Investigational Device Exempt due to low risk of the device). Written consent forms and HIPAA forms were obtained from all the subjects. The recruitment and studies were carried out at 2 clinical sites across multiple weeks of imaging (based on how often the subject returned to the clinic). Hence, images for the current study are only from the first week of the subject's visit and the last week of their visit to the clinic. The wounds are treated as new wounds between the same subject's first week and last week visit (from our 8-week study), because the wounds could 
have physiologically change across weeks of treatment. Thus, a total of 10 DFUs (7 healing and 3 non-healing) were imaged across the 3 subjects (Table 1). Prior to imaging studies, the podiatrists diagnosed the current status of the wound (as healing or non-healing on the day of the visit). The clinical assessment of wounds was by visual inspection of the wound for surface epithelization, and measurements of wound size and depth over weeks, and healing asses from assessed from decrease in wound size over time $(\sim 40 \%$ in 4 weeks of the treatment).

Table 1: Details of the 10 diabetic foot ulcers across 3 subjects imaged across weeks using NIROS.

\begin{tabular}{|c|c|c|c|}
\hline Subject & Week & Wound & $\begin{array}{l}\text { Clinical Diagnosis by Podiatric } \\
\text { Surgeon }\end{array}$ \\
\hline \multirow{6}{*}{$\begin{array}{c}1 \\
56 \text { years old Male }\end{array}$} & \multirow{3}{*}{1} & 1 & \multirow{6}{*}{$\begin{array}{l}\text { Neuropathic, with no arterial } \\
\text { deficiency. All wound sizes } \\
\text { decreased over the weeks, diag- } \\
\text { nosing them as healing wounds. }\end{array}$} \\
\hline & & 2 & \\
\hline & & 3 & \\
\hline & \multirow{3}{*}{5} & 1 & \\
\hline & & 2 & \\
\hline & & 3 & \\
\hline \multirow[t]{2}{*}{$\begin{array}{c}2 \\
70 \text { years old Male }\end{array}$} & 1 & 1 & \multirow{2}{*}{$\begin{array}{l}\text { Neuropathic, with signs of } \\
\text { arterial deficiency. Wound size } \\
\text { did not change over the weeks, } \\
\text { diagnosing it as non- healing } \\
\text { wound. }\end{array}$} \\
\hline & 5 & 1 & \\
\hline \multirow[b]{2}{*}{$\begin{array}{c}3 \\
41 \text { years old Male }\end{array}$} & & 1 & \multirow{2}{*}{$\begin{array}{l}\text { Wound } 1 \text { was a healing wound } \\
\text { and wound } 2 \text { was a non-heal- } \\
\text { ing wound, based on surface } \\
\text { epithelization and past history } \\
\text { of the patient. }\end{array}$} \\
\hline & & 2 & \\
\hline
\end{tabular}

\section{Data Acquisition and Analysis}

Optical imaging was performed on each of the 10 DFUs under rest conditions. Prior to imaging, the wound region was cleaned and debrided (as required) by the podiatric surgeon. The imaging area was chosen such that the wound site and its surrounding region are within the field of view. Diffuse reflectance images were acquired under rest conditions at $10-\mathrm{Hz}$ frequency for 2 seconds (i.e. a total of 20 frames) at 830-nm wavelength. Imaging of each wound was carried out at 2-3 random locations of the optical scanner with respect to the wound and its background. The multiple imaging locations were chosen to determine if the positioning of the scanner with respect to the wound impacted the detected NIR images (which in turn would be used to differentiate a wound as healing or not). White light images (i.e. images under visible light) were also acquired for each wound using the endoscopic camera. Image analyses were performed as described in the following sections.

\section{Data Analysis: Optical Contrast}

A systematic approach was carried out to determine the diffuse reflectance based optical contrasts across a 2-D region of each wound as compared to its periphery (denoted as background). Initially, the detected diffuse reflected NIR signals across the 20 frames were averaged for each wound (termed as 'mean NIR intensity'). The region of interest (ROI) encompassing the wound and the surrounding background tissue was selected from the field of view of the detected NIR signals. Within this ROI, the region encompassing the wound (ROIW) was selected based on the wound location observed from the white light image. The background was selected from the immediate peripheries of the wound (ROIB). The background was selected thrice at different locations around the wound, either encompassing the entire wound or just a small area in the periphery of the wound. If the chosen background encompassed the entire wound, then the intensity in the ROIW region was excluded in calculating the total intensity of ROIB. The background signal may be contaminated by specular reflection and/or non-uniform source strength. Hence to assess the effect of varying signal strengths on the wound: background optical contrast (and eventually in differentiating a wound as healing or not), three different background regions were chosen. The optical contrast was determined as the mean reflected NIR intensity (averaged across all frames) in ROIW with respect to that in ROIB (as shown in equation 1). A Weber-based optical contrast was employed for each case of the background as shown below

$$
\mathrm{W}: \mathrm{B} \text { Contrast }=\frac{\left[\left(\mathrm{ROI}_{\mathrm{w}}\right) \text { mean }-\left(\mathrm{ROI}_{\mathrm{B}}\right) \mathrm{mean}^{*} 100\right.}{\left(\mathrm{ROI}_{\mathrm{B}}\right) \text { mean }}
$$

The entire data analysis process was carried out by two operators (research students) in order to determine if data analysis was operator dependent or independent (statistically evaluated in the following section). It contributes to the robustness of NIROS and the imaging approach by demonstrating that the optical contrasts are operator-independent. The choice of three different backgrounds and two operators was carried out on each wound and each of the location from which it was imaged. The optical contrast ratios were determined for all the subjects' wounds and depicted as scatter plots to compare the differences between healing and non-healing wounds. Thus a total of 7 healing DFUs and 3 non-healing DFUs were evaluated to yield three optical contrasts for each imaging location and repeated by 2 operators (or) researchers in the above studies (i.e. a total of 162 combination of processed images).

\section{Results and Discussion}

\section{Optical Contrast Studies}

The white light and detected NIR images are shown along with the reflected W:B optical contrast values in Figure 2 for one case of a healing and non-healing DFU. The healing DFU showed a positive W:B optical contrast and a non-healing DFU showed a W:B negative optical contrast. A scatter plot of all the optical contrasts obtained from 7 healing and 3 non-healing wounds under various imaging conditions (see section 2.C.1) are given in Figure 3. A distinct difference in the optical contrasts was observed between healing and non-healing wounds. While all healing wounds (except a few cases) had a positive diffuse optical contrast, the non- healing wounds had a negative diffuse optical contrast. Average optical contrast measurement ( $\mathrm{n}=48$ images) for non-healing wounds was -34.27 (SD: 24.08). For healing wounds, the average optical contrast measurement ( $\mathrm{n}=114$ images) were 24.43 (SD: 22.81). The difference between the optical contrast for non-healing and healing wounds was estimated to be -58.70 (95\% CI: - 66.58,-50.81). This implies that there is a greater absorption of NIR light in a non-healing wound compared to a healing wound. The extent of absorption or reflec- 
tion of NIR light is directly proportional to the concentration of the major tissue components (e.g. HbO, HbR, water) [36]. A normal wound (or ulcer) progresses through various stages of healing including hemostasis, inflammatory, proliferation, and remodeling [37-39]. A non-healing VLU remains at the inflammatory phase, during which there is cessation of epidermal growth and migration over the wound surface (that elevate the HbR concentrations at the wound site). On the contrary, a healing wound progress to other phases, where there is lesser HbR due to cell growth [12,13,25]. NIR absorption of light at 830- $\mathrm{nm}$ increases (or its diffuse reflectance decreases) with increased HbR concentrations, as this wavelength is sensitive to the changes in concentration of HbR [22]. Thus, a decreased diffuse reflectance from the wound in comparison to its background causes a negative (diffuse reflectance based) optical contrast in non-healing wounds.

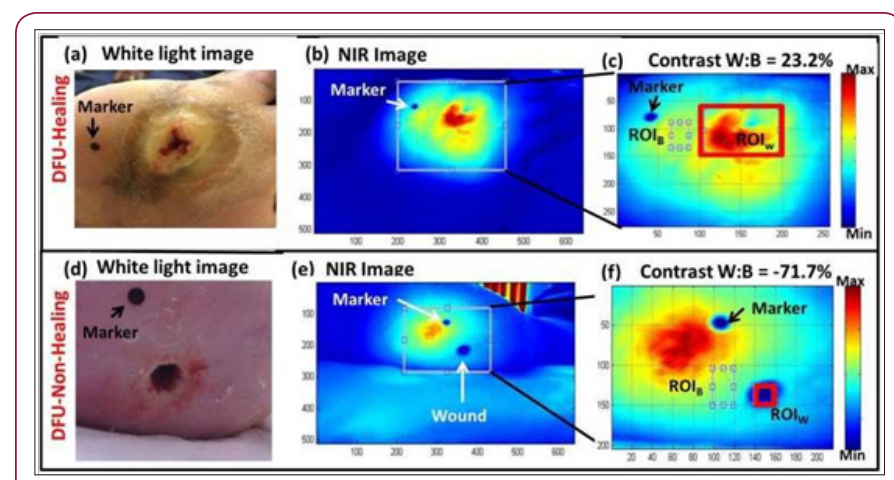

Figure 2: White light and NIR (diffuse reflectance) images of a healing and non-healing DFU showing region of interest (ROI) of the wound (W) and background (B) selected in order to estimate the NIR optical contrast.

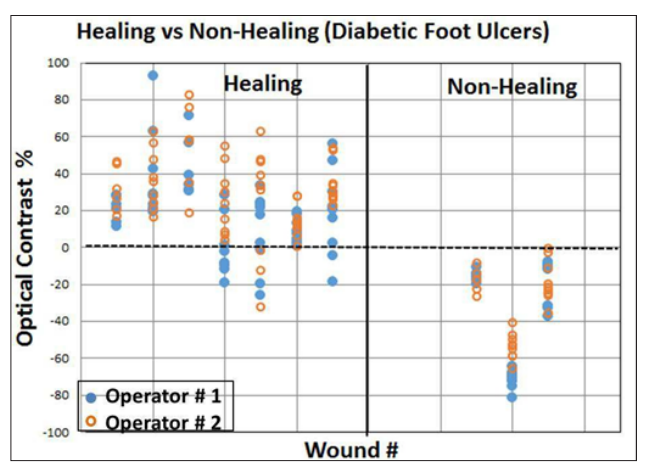

Figure 3: Scatter plot of NIR optical contrast obtained from 7 healing and 3 non-healing DFUs, imaged at multiple locations per wound, chose ROIB three different ways, and analyzed by two independent operators.

In the current studies, all the non-healing DFUs were from subjects that the podiatrist has been treating or assessing for many months to a year. Hence, the clinical baseline was confirmed prior to correlating the W:B optical contrasts with the healing or non-healing status of the wounds. Future studies will involve blinded imaging studies of wounds without a prior knowledge of their status from the clinician, and comparing the effectiveness of NIROS in differentiating them as healing or non-healing. Sensitivity and specificity analysis of NIROS can positively impact objective wound assessment based on the underlying physiology of the wound and not just its surface visual assessment (which is the current clinical practice). In addition, the effect of skin color in and around the wound will be recorded and assessed to determine its impact on the optical contrast between the wound and background.

\section{Conclusion}

In summary, an ultra-portable handheld near-infrared optical scanner (NIROS) has been implemented to perform non-contact imaging of diabetic foot ulcers (DFUs). Preliminary studies demonstrated that NIROS was capable of differentiating healing from non-healing wounds based on differences in the optical contrast between the wound and its peripheries. In the current study, the robustness of NIROS to variations in its location during imaging, to the choice of background regions during image analysis and operator dependency have been demonstrated from clinical studies on DFUs. Currently, the device is modified such that it can acquire oxy(HbO) and deoxy-hemoglobin ( $\mathrm{HbR}$ ) concentration changes of the wound with respect to its peripheries, and also monitor healing of the wound periodically over a few weeks. In future, imaging studies will be carried using NIROS to assess the changes in hemodynamics of the entire wound area across weeks. In addition, with the advantage of non-contact wide-field imaging capabilities of NIROS, the entire area of the wound can be imaged. This allows segmentation of regions with varied blood oxygenation in and around the visual wound, to potentially detect the effectiveness of the treatment to promote oxygenation to the wound that is crucial for healing.

\section{Acknowledgment}

The authors would like to thank FIU CEC-BME Seed Funds for the funding support. One of the authors would like to acknowledge NIH-MARC U*STAR Program (T34 GM083688) for funding support.

\section{References}

1. Centers for Disease Control and Prevention (2014) National Diabetes Statistics Report: Estimates of Diabetes and Its Burden in the United States, U.S. Department of Health and Human Services, Atlanta, Georgia.

2. Rice JB, Desai U, Cummings AK (2014) Burden of diabetic foot ulcers for medicare and private insurers. Diabetes Care 37: 651-658.

3. Wild S, Roglic G, Green A, Sicree R, King H (2004) Global prevalence of diabetes: estimates for the year 2000 and projections for 2030. Diabetes Care 27(3): 1047-1053.

4. Lavery LA, Armstrong DG, Wunderlich RP, Mohler MJ, Wendel CS, et al. (2006) Risk factors for foot infections in individuals with diabetes. Diabetes Care 29(6): 1288-1293.

5. Pecoraro RE, Reiber GE, Burgess EM (1990) Pathways to diabetic limb amputation: Basis for prevention. Diabetes Care 13(5): 513-521.

6. Singh N, Armstrong DG, Lipsky BA (2005) Preventing foot ulcers in patients with diabetes. JAMA

7. 293(2): 217-218.

8. American Diabetes Association (2013) Economic costs of diabetes in the U.S. in 2012. Diabetes Care 36(4): 1033-1046.

9. Sen CK, Gordillo GM, Roy S (2009) Human skin wounds: a major and snowballing threat to public health and the economy. Wound Repair Regeneration 17: 763-771.

10. Kruse I, Edelman S (2006) Evaluation and treatment of diabetic foot ulcers. Clinical Diabetes 24(2): 91-93. 
11. Ragnarson Tennvall G, Apelqvist J (2004) Health-Economic Consequences of Diabetic Foot Lesions. Clinical Infectious Diseases 39(Suppl 2): S132-S139.

12. Steed DL, Attinger C, Colaizzi T (2006) Guidelines for the treatment of diabetic ulcers. Wound Repair Regen 14(16): 680-692.

13. Neidrauer M, Zubkov L, Weingarten MS, Pourrezaei K, Papazoglou ES (2010) Near infrared wound monitor helps clinical assessment of diabetic foot ulcers. J Diabetes Sci Technol 4(4): 792-798.

14. Weingarten MS, Neidrauer M, Mateo A (2010) Prediction of wound healing in human diabetic foot ulcers by diffuse near-infrared spectroscopy: A pilot study. Wound Rep Regen 18(2): 180-185.

15. Boulton AJ, Vileikyte L, Ragnarson Tennvall G, Apelqvist J (2005) The global burden of diabetic foot disease. Lancet 366(9498): 1719-1724.

16. Frykberg RG (2002) Diabetic foot ulcers: pathogenesis and management. Am Fam Physician 66(9): 1655-1662.

17. Zimny S, Schatz H, Pfohl M (2004) The effects of ulcer size on the wound radius reductions and healing times in neuropathic diabetic foot ulcers. Exp Clin Endocrinol Diabetes 112(4): 191-194.

18. Margolis DJ, Gelfand JM, Hoffstad O, Berlin JA (2003) Surrogate end points for the treatment of diabetic neuropathic foot ulcers. Diabetes Care 26(6): 1696-1700.

19. Falanga $V$ (2005) Wound healing and its impairment in the diabetic foot. Lancet 366(9498): 1736-1743.

20. Mackaay AJ, Beks PJ, Dur AH (1995) The distribution of peripheral vascular disease in a Dutch Caucasian population: comparison of type II diabetic and non-diabetic subjects. Eur J Vasc Endovasc Surg 9(2): 170175.

21. Vowden PVK (2001) The management of diabetic foot ulcers. Martin Dunitz, London.

22. Pecoraro RE, Ahroni JH, Boyko EJ, Stensel VL (1991) Chronology and determinants of tissue repair in diabetic lower-extremity ulcers. Diabetes 40(10): 1305-1313.

23. Paul DW, Ghassemi P, Ramella Roman JC (2015) Noninvasive imaging technologies for cutaneous wound assessment: A review. Wound Rep Regen 23(2): 149-162.

24. Jayachandran M, Rodriguez S, Solis E, Lei J, Godavarty A (2016) Critical review of noninvasive optical technologies for wound imaging. Adv Wound Care (Invited Review) 5(8): 349-359.

25. Papazoglou ES, Weingarten MS, Zubkov L, Neidrauer M, Pourrezaei K (2008) Assessment of diabetic foot ulcers with diffuse near infrared methodology. $8^{\text {th }}$ IEEE International Conference Bioinforma, Bioeng, p. $1-5$.

26. Papazoglou ES, Neidrauer M, Zubkov L, Weingarten MS, Pourrezaei K (2009) Noninvasive assessment of diabetic foot ulcers with diffuse photon density wave methodology: pilot human study. J Biomed Opt 14(6): 064032.

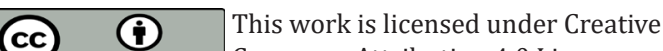

Submission Link: https://biomedres.us/submit-manuscript.php
27. Jung YJ, Roman M, Carrasquilla J, Erickson SJ, Godavarty A (2014) Portable wide-field hand-held NIR scanner. SPIE Proceedings 8572: 85720A.

28. Jung YJ, Gonzalez J, Rodriguez S, Mejia MV, Clark G, et al. (2014) Anatomical Co- Registration using Spatio-Temporal Features of a Noncontact Near-Infrared Optical Scanner. SPIE Proceedings 89420: 1-5.

29. Moza R, Dimaio JM, Melendez J (2010) Deep-Tissue Dynamic Monitoring of Decubitus Ulcers: Wound Care and Assessment. IEEE Eng Med Biol Mag 29(2): 71-77.

30. Weingarten MS, Samuels JA, Neidrauer M (2012) Diffuse near-infrared spectroscopy prediction of healing diabetic foot ulcers: A human study and cost analysis. Wound Repair Regen 20(6): 911-917.

31. Papazoglou ES, Zubkov L, Zhu L, Weingarten MS, Tyagi S, et al. (2005) Monitoring Diabetic Wound Healing By NIR Spectroscopy. Conf Proc IEEE Eng Med Biol Sco 4: 6662-6664.

32. Papazoglou E, Weingarten M, Zubkov L, Zhu, S Tyagi L, et al. (2007) Near Infrared Diffuse Optical Tomography: Improving the Quality of Care In Chronic Wounds Of Patients With Diabetes. Biomed Inst Tech 41(1): 8387.

33. Papazoglou ES, Weingarten MS, Zubkov L, Zhu L, Tyagi S, et al. (2006) Optical Properties of Wounds: Diabetic Versus Healthy Tissue. IEEE Trans Biomed Eng 53(6): 1047-1055.

34. Anand S, Sujatha N, Narayanamurthy VB, Seshadri V, Poddar R (2014) Diffuse Reflectance Spectroscopy for Monitoring Diabetic Foot Ulcer - A Pilot Study. Optics and Lasers in Engineering 53: 1-5.

35. Godavarty A, Khandavilli Y, Jung YJ, Rao PN (2015) Non-contact optical imaging of healing and non-healing diabetic foot ulcers. Proc SPIE, pp. 931802-931802.

36. Godavarty A, Rao PN, Khandavilli Y, Jung YJ (2015) Diabetic wound imaging using a non- contact near-infrared scanner: A pilot study. J Diabetes Sci Technol 9(5): 1158-1159.

37. Wray S, Cope M, Delpy DT, Wyatt JS, Reynolds EO (1989) Characterization of the near infrared absorption spectra of cytochrome aa3 and hemoglobin for the non-invasive monitoring of cerebral oxygenation. Biochem Biophys Acta 933(1): 184-192.

38. Singer A, Clark RA (1999) Cutaneous wound healing. N Engl J Med 341(10): 738-746.

39. Gurtner G, Werner S, Barrandon Y, Longaker MT (2008) Wound repair and regeneration. Nature

40. 453: 314-321.

41. Schreml S, Szeimies RM, Prantl L, Landthaler M, Babilas P (2010) Wound healing in the $21^{\text {st }}$ century. J Am Acad Dermatol 63(5): 866-888.

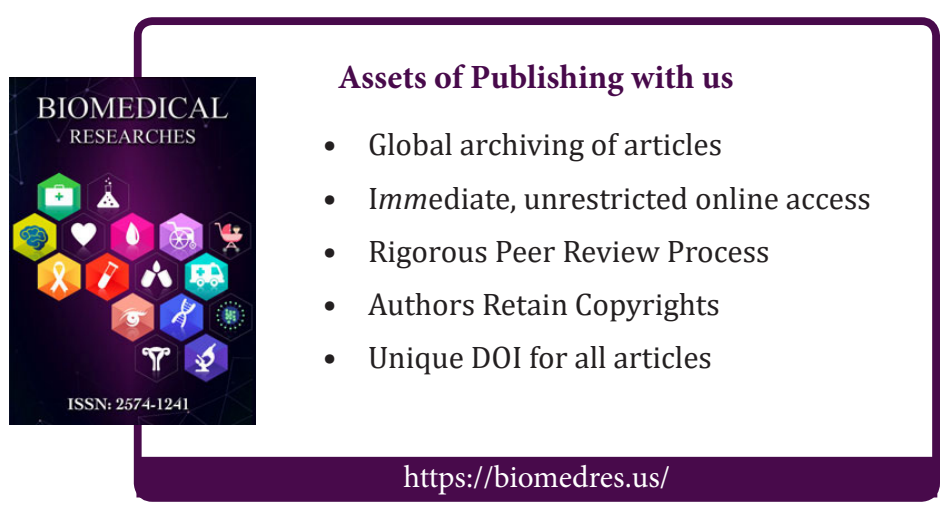

